\title{
Erratum to: Subclavian Vein Versus Arm Vein for Totally Implantable Central Venous Port for Patients with Head and Neck Cancer: A Retrospective Comparative Analysis
}

\author{
Akio Akahane - Miyuki Sone - Shigeru Ehara • \\ Kenichi Kato - Ryoichi Tanaka - Tatsuhiko Nakasato
}

Published online: 26 May 2011

(C) Springer Science+Business Media, LLC and the Cardiovascular and Interventional Radiological Society of Europe (CIRSE) 2011

Erratum to: Cardiovasc Intervent Radiol

DOI 10.1007/s00270-010-0051-4

This erratum is being published to correct the error of the article entitled "Subclavian Vein Versus Arm Vein for Totally Implantable Central Venous Port for Patients with Head and Neck Cancer: A Retrospective Comparative Analysis" DOI:10.1007/s00270-010-0051-4, Editorial manuscript number: CVR-2010-0478.R2, as follows. Table 3 of the summary of adverse event should read as follows.

The online version of the original article can be found under doi: 10.1007/s00270-010-0051-4.

\footnotetext{
A. Akahane $(\varangle) \cdot$ M. Sone $\cdot$ S. Ehara $\cdot$ K. Kato $\cdot$ R. Tanaka T. Nakasato

Department of Radiology, Iwate Medical University School of Medicine, 19-1 Uchimaru, Morioka 020-8505, Japan

e-mail: a.akahane@gmail.com
} 
Table 3 Adverse events

\begin{tabular}{|c|c|c|c|c|c|}
\hline \multirow[t]{2}{*}{ Adverse event } & \multicolumn{2}{|c|}{ Subclavian ports } & \multicolumn{2}{|l|}{ Arm ports } & \multirow[t]{2}{*}{$P$ Value } \\
\hline & No. $(\%)$ & $\begin{array}{l}\text { Median } \\
\text { duration (days) }\end{array}$ & No. $(\%)$ & $\begin{array}{l}\text { Median } \\
\text { duration (days) }\end{array}$ & \\
\hline \multicolumn{6}{|l|}{ Periprocedural } \\
\hline Pneumothorax & $1(2.1)$ & & $0(0)$ & & 0.290 \\
\hline Arterial puncture & $1(2.1)$ & & $0(0)$ & & 0.290 \\
\hline Primary malposition & $0(0)$ & & $0(0)$ & & \\
\hline Total & $2(4.3)$ & & $0(0)$ & & 0.830 \\
\hline \multicolumn{6}{|l|}{ Postprocedural } \\
\hline Infection** & $4(8.5)$ & 49 & $9(7.8)$ & 44.5 & 0.758 \\
\hline Phlebitis & $0(0)$ & & $11(9.5)$ & 33 & $0.035^{*}$ \\
\hline Fibrin sheath & $0(0)$ & & $0(0)$ & & \\
\hline System occlusion & $0(0)$ & & $5(4.3)$ & 53 & 0.323 \\
\hline Subcutaneous extravasation & $0(0)$ & & $1(0.9)$ & 34 & 1.000 \\
\hline Venous thrombosis & $0(0)$ & & $0(0)$ & & \\
\hline Catheter detachment & $0(0)$ & & $0(0)$ & & \\
\hline Total & $4(8.5)$ & 49 & $26(22.6)$ & 66 & $0.044 *$ \\
\hline
\end{tabular}

CVP central venous port

$* P<0.05$

** Details of infectious AEs are listed in Table 4 